\title{
Drawing Planar Graphs with a Prescribed Inner Face
}

\author{
Tamara Mchedlidze, Martin Nöllenburg, and Ignaz Rutter
}

Institute of Theoretical Informatics, Karlsruhe Institute of Technology (KIT), Germany

\begin{abstract}
Given a plane graph $G$ (i.e., a planar graph with a fixed planar embedding) and a simple cycle $C$ in $G$ whose vertices are mapped to a convex polygon, we consider the question whether this drawing can be extended to a planar straight-line drawing of $G$. We characterize when this is possible in terms of simple necessary conditions, which we prove to be sufficient. This also leads to a linear-time testing algorithm. If a drawing extension exists, it can be computed in the same running time.
\end{abstract}

\section{Introduction}

The problem of extending a partial drawing of a graph to a complete one is a fundamental problem in graph drawing that has many applications, e.g., in dynamic graph drawing and graph interaction. This problem has been studied most in the planar setting and often occurs as a subproblem in the construction of planar drawings.

The earliest example of such a drawing extension result are so-called Tutte embeddings. In his seminal paper "How to Draw a Graph" [10], Tutte showed that any triconnected planar graph admits a convex drawing with its outer vertices fixed to an arbitrary convex polygon. The strong impact of this fundamental result is illustrated by its, to date, more than 850 citations and the fact that it received the "Best Fundamental Paper Award” from GD'12. The work of Tutte has been extended in several ways. In particular, it has been strengthened to only require polynomial area [4], even in the presence of collinear points [3]. Hong and Nagamochi extended the result to show that triconnected graphs admit convex drawings when their outer vertices are fixed to a star-shaped polygon [5]. For general subdrawings, the decision problem of whether a planar straight-line drawing extension exists is NP-hard [9]. Pach and Wenger [8] showed that every subdrawing of a planar graph that fixes only the vertex positions can be extended to a planar drawing with $O(n)$ bends per edge and that this bound is tight. The drawing extension problem has also been studied in a topological setting, where edges are represented by non-crossing curves. In contrast to the straight-line variant, it can be tested in linear time whether a drawing extension of a given subdrawing exists [1]. Moreover, there is a characterization via forbidden substructures [6].

In this paper, we study the problem of finding a planar straight-line drawing extension of a plane graph for which an arbitrary cycle has been fixed to a convex polygon. It is easy to see that a drawing extension does not always exist in this case; see Fig. 1(a), Let $G$ be a plane graph and let $C$ be a simple cycle of $G$ represented by a convex polygon $\Gamma_{C}$ in the plane. The following two simple conditions are clearly necessary for the existence of a drawing extension: (i) $C$ has no chords that must be embedded outside of $C$ and (ii) for every vertex $v$ with neighbors on $C$ that must be embedded outside of

S. Wismath and A. Wolff (Eds.): GD 2013, LNCS 8242, pp. 316-327, 2013.

(C) Springer International Publishing Switzerland 2013 
$C$ there exists a placement of $v$ outside $\Gamma_{C}$ such that the drawing of the graph induced by $C$ and $v$ is plane and bounded by the same cycle as in $G$. We show in this paper that these two conditions are in fact sufficient. Both conditions can be tested in linear time, and if they are satisfied, a corresponding drawing extension can be constructed within the same time bound.

Our paper starts with some necessary definitions (Section 2) and useful combinatorial properties (Section 3). The idea of our main result has two steps. We first show in Section 4 that the conditions are sufficient if $\Gamma_{C}$ is one-sided (i.e., it has an edge whose incident inner angles are both less than $90^{\circ}$ ). Afterward, we show in Section 5 that, for an arbitrary convex polygon $\Gamma_{C}$, we can place the neighborhood of $C$ in such a way that the drawing is planar, and such that the boundary $C^{\prime}$ of its outer face is a one-sided polygon $\Gamma_{C^{\prime}}$. Moreover, our construction ensures that the remaining graph satisfies the conditions for extendability of $\Gamma_{C^{\prime}}$. The general result then follows directly from the one-sided case.

\section{Definitions and a Necessary Condition}

Plane Graphs and Subgraphs. A graph $G=(V, E)$ is planar if it has a drawing $\Gamma$ in the plane $\mathbb{R}^{2}$ without edge crossings. Drawing $\Gamma$ subdivides the plane into connected regions called faces; the unbounded region is the outer and the other regions are the inner faces. The boundary of a face is called facial cycle, and outer cycle for the outer face. The cyclic ordering of edges around each vertex of $\Gamma$ together with the description of the external face of $G$ is called an embedding of $G$. A graph $G$ with a planar embedding is called plane graph. A plane subgraph $H$ of $G$ is a subgraph of $G$ together with a planar embedding that is the restriction of the embedding of $G$ to $H$.

Let $G$ be a plane graph and let $C$ be a simple cycle of $G$. Cycle $C$ is called strictly internal, if it does not contain any vertex of the outer face of $G$. A chord of $C$ is called outer if it lies outside $C$ in $G$. A cycle without outer chords is called outerchordless. The subgraph of $G$ inside $C$ is the plane subgraph of $G$ that is constituted by vertices and edges of $C$ and all vertices and edges of $G$ that lie inside $C$.

Connectivity. A graph $G$ is $k$-connected if removal of any set of $k-1$ vertices of $G$ does not disconnect the graph. For $k=2,3$ a $k$-connected graph is also called biconnected and triconnected, respectively. An internally triangulated plane graph is triconnected if and only if there is no edge connecting two non-consecutive vertices of its outer cycle (see, for example, [2]).

Star-shaped and One-sided Polygons. Let $\Pi$ be a polygon in the plane. Two points inside or on the boundary of $\Pi$ are mutually visible, if the straight-line segment connecting them belongs to the interior of $\Pi$. The kernel $K(\Pi)$ of polygon $\Pi$ is the set of all the points inside $\Pi$ from which all vertices of $\Pi$ are visible. We say that $\Pi$ is star-shaped if $K(\Pi) \neq \emptyset$. We observe that the given definition of a star-shape ensures that its kernel has a positive area.

A convex polygon $\Pi$ with $k$ vertices is called one-sided, if there exists an edge $e$ (i.e., a line segment) of $\Pi$ such that the orthogonal projection to the line supporting $e$ 


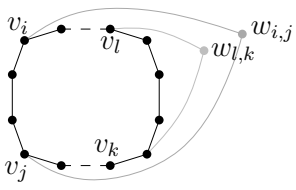

(a)

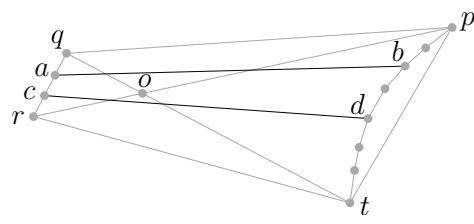

(b)

Fig. 1. Convex polygon of cycle $C$ is denoted by black. Vertex $w_{i, j}$ cannot be placed on the plane without changing the embedding or intersecting $C$. Vertices $w_{i, j}$ and $w_{l, k}$ are petals of $C$, where $w_{l, k} \prec w_{i, j}$. Petal $w_{l, k}$ is realizable, while petal $w_{i, j}$ is not. (b) Illustration of Fact 1 .

maps all polygon vertices actually onto segment $e$. Then $e$ is called the base edge of $\Pi$. Without loss of generality let $e=\left(v_{1}, v_{k}\right)$ and $v_{1}, \ldots, v_{k}$ be the clockwise ordered sequence of vertices of $\Pi$.

Extension of a Drawing. Let $G$ be a plane graph and let $H$ be a plane subgraph of $G$. Let $\Gamma_{H}$ be a planar straight-line drawing of $H$. We say that $\Gamma_{H}$ is extendable if drawing $\Gamma_{H}$ can be completed to a planar straight-line drawing $\Gamma_{G}$ of the plane graph $G$. Then $\Gamma_{G}$ is called an extension of $\Gamma_{H}$. A planar straight-line drawing of $G$ is called convex, if every face of $G$ (including the outer face) is represented as a convex polygon.

The following theorem by Hong and Nagamochi [5] shows the extendability of a prescribed star-shaped outer face of a plane graph.

Theorem 1 (Hong, Nagamochi [5]). Every drawing of the outerface f of a 3-connected graph $G$ as a star-shaped polygon can be extended to a planar drawing of $G$, where each internal face is represented by a convex polygon. Such a drawing can be computed in linear time.

Petals and Stamens. Let $G$ be a plane graph, and let $P_{u v}$ be a path in $G$ between vertices $u$ and $v$. Its subpath from vertex $a$ to vertex $b$ is denoted by $P_{u v}[a, b]$. Let $C$ be a simple cycle of $G$, and let $v_{1}, \ldots, v_{k}$ be the vertices of $C$ in clockwise order. Given two vertices $v_{i}$ and $v_{j}$ of $C$, we denote by $C\left[v_{i}, v_{j}\right]$ the subpath of $C$ encountered when we traverse $C$ clockwise from $v_{i}$ to $v_{j}$. Assume that $C$ is represented by a convex polygon $\Gamma_{C}$ in the plane. We say that a vertex $v_{i}, 1 \leq i \leq k$ of $\Gamma_{C}$ is flat, if $\angle v_{i-1} v_{i} v_{i+1}=\pi$. Throughout this paper, we assume that convex polygons do not have flat vertices.

A vertex $w \in V(G) \backslash V(C)$ adjacent to at least two vertices of $C$ and lying outside $C$ in $G$, is called a petal of $C$ (see Figure 1(a). Consider the plane subgraph $G^{\prime}$ of $G$ induced by the vertices $V(C) \cup\{w\}$. Vertex $w$ appears on the boundary of $G^{\prime}$ between two vertices of $C$, i.e. after some $v_{i} \in V(C)$ and before some $v_{j} \in V(C)$ in clockwise order. To indicate this fact, we will denote petal $w$ by $w_{i, j}$. Edges $\left(w_{i, j}, v_{i}\right)$ and $\left(w_{i, j}, v_{j}\right)$ are called the outer edges of petal $w_{i, j}$. The subpath $C\left[v_{i}, v_{j}\right]$ of $C$ is called base of the petal $w_{i, j}$. A vertex $v_{f}$ is called internal, if it appears on $C$ after $v_{i}$ and before $v_{j}$ in clockwise order. A petal $w_{i, i+1}$ is called trivial. A vertex of $V(G) \backslash V(C)$ adjacent to exactly one vertex of $C$ is called a stamen of $C$.

Let $v$ be a petal of $C$ and let $u$ be either a petal or a stamen of $C$, we say that $u$ is nested in $v$, and denote this fact by $u \prec v$, if $u$ lies in the cycle delimited by the base 
and the outer edges of petal $v$. For two stamens $u$ and $v$, neither $u \prec v$ nor $v \prec u$. So for each pair of stamens or petals $u$ and $v$ we have either $u \prec v$, or $v \prec u$, or none of these. This relation $\prec$ is a partial order. A petal or a stamen $u$ of $C$ is called outermost if it is maximal with respect to $\prec$.

Necessary Petal Condition. Let again $G$ be a plane graph and let $C$ be an outerchordless cycle of $G$ represented by a convex polygon $\Gamma_{C}$ in the plane. Let $w_{i, j}$ be a petal of $C$. Let $G^{\prime}$ be the plane subgraph of $G$, induced by the vertices $V(C) \cup\left\{w_{i, j}\right\}$. We say that $w_{i, j}$ is realizable if there exists a planar drawing of $G^{\prime}$ which is an extension of $\Gamma_{C}$. This gives us the necessary condition that $\Gamma_{C}$ is extendable only if each petal of $C$ is realizable. In the rest of the paper we prove that this condition is sufficient.

\section{Combinatorial Properties of Graphs and Petals}

In this section, we derive several properties of petals in graphs, which we use throughout the construction of the drawing extension in the remaining parts of this paper. Due to space constraints the proofs can be found in the full version of this paper [7]. Proposition 1 allows us to restrict our attention to maximal plane graphs for which the given cycle $C$ is strictly internal. The remaining lemmas are concerned with the structure of the (outermost) petals of $C$ in such a graph.

Proposition 1. Let $G$ be a plane graph on $n$ vertices and let $C$ be a simple outerchordless cycle of $G$. There exists a plane supergraph $G^{\prime}$ of $G$ with $O(n)$ vertices such that (i) $G^{\prime}$ is maximal, (ii) there are no outer chords of $C$ in $G^{\prime}$, (iii) each petal of $G^{\prime}$ with respect to $C$ is either trivial or has the same neighbors on $C$ as in $G$, and (iv) $C$ is strictly internal to $G$.

In the following we assume that our given plane graph is maximal, and the given cycle is strictly internal, otherwise Proposition 1 is applied.

Lemma 1. Let $G$ be a triangulated planar graph with a strictly internal outerchordless cycle $C$. Then the following statements hold. $(i)$ Each vertex of $C$ that is not internal to an outermost petal is adjacent to two outermost petals. (ii) There is a simple cycle $C^{\prime}$ whose interior contains $C$ and that contains exactly the outermost stamens and petals of $C$.

Lemma 2. Let $G$ be a maximal planar graph with a strictly internal outerchordless cycle $C$. Let $u$ and $v$ be two adjacent vertices on $C$ that are not internal to the same petal. Then there exists a third vertex $w$ of $C$ such that there exist three chordless disjoint paths from $u, v$ and $w$ to the vertices of the outer face of $G$ such that none of them contains other vertices of $C$.

\section{Extension of a One-sided Polygon}

Let $G$ be a plane graph, and let $C$ be a simple outerchordless cycle, represented by a one-sided polygon $\Gamma_{C}$. In this section, we show that if each petal of $C$ is realizable, 
then $\Gamma_{C}$ is extendable to a straight-line drawing of $G$. This result serves as a tool for the general case, which is shown in Section 5

The drawing extension we produce preserves the outer face, i.e., if the extension exists, then it has the same outer face as $G$. It is worth mentioning that, if we are allowed to change the outer face, the proof becomes rather simple, as the following claim shows.

Claim 1. Let $G$ be a maximal plane graph and let $C$ be an outerchordless cycle of $G$, represented in the plane by a one-sided polygon $\Gamma_{C}$. Then drawing $\Gamma_{C}$ is extendable.

Proof. Let $\left(v_{1}, v_{k}\right)$ be the base edge of $\Gamma_{C}$. Edge $\left(v_{1}, v_{k}\right)$ is incident to two faces of $G$, to a face $f_{\text {in }}$ inside $C$ and to a face $f_{\text {out }}$ outside $C$. We select $f_{\text {out }}$ as the outer face of $G$. With this choice, edge $\left(v_{1}, v_{k}\right)$ is on the outer face of $G$. Let $v$ be the third vertex of this face. We place the vertex $v$ far enough from $\Gamma_{C}$, so that all vertices of $\Gamma_{C}$ are visible from $v$. Thus, we obtain a planar straight-line drawing of the subgraph $G_{v}$ induced by the vertices $V(C) \cup\{v\}$, such that each face is represented by a star-shaped polygon. Each subgraph of $G$ inside a face of $G_{v}$ is triconnected, and therefore, we can complete the existing drawing to a straight-line planar drawing of $G$, by Theorem 1 .

In the rest of the section we show that extendability of $\Gamma_{C}$ can be efficiently tested, even if the outer face of $G$ has to be preserved. The following simple geometric fact will be used in the proof of the result of this section (see Figure 1(b) for the illustration).

Fact 1. Let pqrt be a convex quadrilateral and let o be the intersection of its diagonals. Let $S_{p t}$ be a one-sided convex polygon with base $\overline{p t}$, that lies inside triangle $\triangle$ opt. Let $\overline{a b}$ and $\overline{c d}$ be such that $b, d \in S_{p t}$, ordered clockwise as $t, d, b, p$ and $a, c \in \overline{q r}$, ordered as $q, a, c, r$. Then, neither $\overline{a b}$ and $\overline{c d}$ intersect each other, nor do they intersect a segment between two consecutive points of $S_{p t}$.

We are now ready to prove the main result of this section.

Theorem 2. Let $G$ be a maximal plane graph and $C$ be a strictly internal simple outerchordless cycle of $G$, represented in the plane by a one-sided polygon $\Gamma_{C}$. If every petal of $C$ is realizable, then $\Gamma_{C}$ is extendable.

Proof. Let $v_{1}, \ldots, v_{k}$ be the clockwise ordering of the vertices of $C$, so that $\left(v_{1}, v_{k}\right)$ is the base of $\Gamma_{C}$. We rotate $\Gamma_{C}$ so that $\left(v_{1}, v_{k}\right)$ is horizontal. Let $a, b, c$ be the vertices of the external face of $G$, in clockwise order, see Fig. 2. By Lemma 2, there exists a vertex $v_{j}, 1<j<k$, such that there exist chordless disjoint paths between $v_{1}, v_{j}, v_{k}$, and the vertices $a, b, c$, respectively. Without loss of generality assume they are $P_{v_{1} a}, P_{v_{j} b}$ and $P_{v_{k} c}$. Some vertices of $P_{v_{1} a}$ and $P_{v_{k} c}$ are possibly adjacent to each other, as well as to the boundary of $C$. Depending on these adjacencies, we show how to draw the paths $P_{v_{1} a}, P_{v_{k} c}$ and how to place vertex $b$, so that the graph induced by these vertices and cycle $C$ is drawn with star-shaped faces. Then, the drawing of $G$ can be completed by applying Theorem 11 Let $v_{i}$ be the topmost vertex of $\Gamma_{C}$. It can happen that there are two adjacent topmost vertices $v_{i}$ and $v_{i+1}$. However, $v_{i-1}$ and $v_{i+2}$ are lower, since $\Gamma_{C}$ does not contain flat vertices. In the following, we assume that $v_{i}$ and $v_{i+1}$ have the same $y$-coordinate. The case when $v_{i}$ is unique can be seen as a special case where $v_{i}=v_{i+1}$. Without loss of generality assume that $i+1 \leq j \leq k-1$, the case where $2 \leq j \leq i$ 


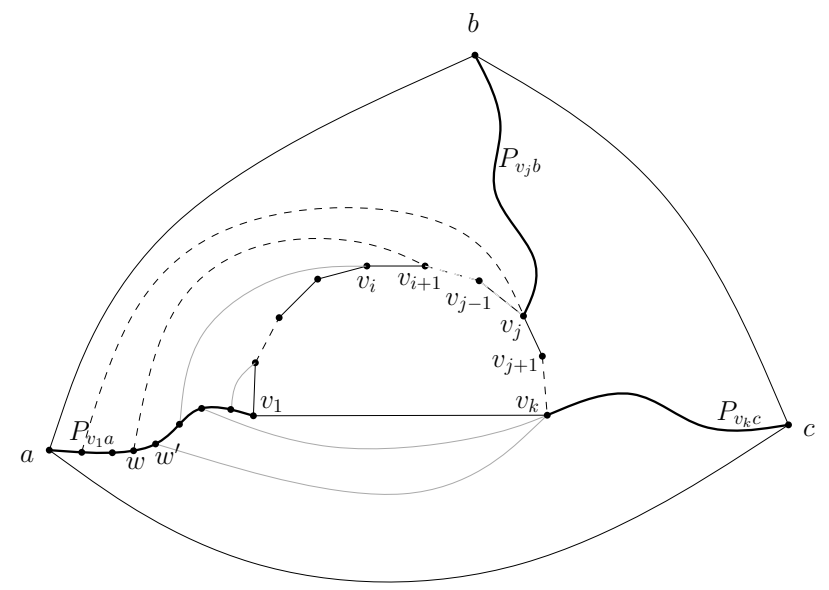

Fig. 2. Illustration for the proof of Theorem 2 Edges between $C\left[v_{1}, v_{i}\right]$ and $P_{v_{1} a}\left[v_{1}, w^{\prime}\right] \cup\left\{v_{k}\right\}$ are gray. Edges between $C\left[v_{i+1}, v_{j}\right]$ and $P_{v_{1} a}[w, a]$ are dashed.

is treated symmetrically. Notice that the presence of the path $P_{v_{j} b}$ ensures that edges between vertices of $P_{v_{1} a}$ and $P_{v_{k} c}$ can only lie in the interior of the cycle delimited by these paths and edges $\left(v_{1}, v_{k}\right)$ and $(a, c)$ (refer to Figure 2). Consider a vertex of $P_{v_{1} a}$ which is a petal of $C$. The base of such a petal cannot contain edge $\left(v_{k-1}, v_{k}\right)$, since this would cause a crossing with $P_{v_{k} c}$. Moreover, if the base of this petal contains edge $\left(v_{1}, v_{k}\right)$, then it cannot contain any edge $\left(v_{f}, v_{f+1}\right)$ for $i \leq f<j$, since otherwise this petal would not be realizable. Thus a vertex of $P_{v_{1} a}$ is either adjacent to $v_{k}$ or to a vertex $v_{f}$, where $i+1 \leq f \leq j$, but not both. It is worth mentioning that a vertex of $P_{v_{1} a}$ cannot be adjacent to any $v_{f}, j+1 \leq f \leq k-1$, since such an adjacency would cause a crossing either with $P_{v_{j} b}$ or with $P_{v_{k} c}$.

Let $\ell_{a}, \ell$ and $\ell_{c}$ be three distinct lines through $v_{j}$ that lie clockwise between the slopes of edges $\left(v_{j-1}, v_{j}\right)$ and $\left(v_{j}, v_{j+1}\right)$ (see Figure 3). Such lines exist since $\Gamma_{C}$ does not contain flat vertices. Let $\ell_{i}$ be the line through $v_{i}$ with the slope of $\left(v_{i-1}, v_{i}\right)$. Let $\ell_{a}^{1}$ be the half-line originating at an internal point of $\left(v_{1}, v_{k}\right)$ towards $-\infty$, slightly rotated counterclockwise from the horizontal position, so that it crosses $\ell_{i}$. Let $q$ denote the intersection point of $\ell_{a}^{1}$ and $\ell_{i}$. Let $p$ be any point on $\ell_{a}^{1}$ further away from $v_{1}$ than $q$. Let $\ell_{a}^{2}$ be the line through $p$ with the slope of $\ell$. By construction of lines $\ell_{a}, \ell$ and $\ell_{c}$, line $\ell_{a}^{2}$ crosses $\ell_{a}$ above the polygon $\Gamma_{C}$ at point $p_{a}$ and line $\ell_{c}$ below this polygon at point $p_{c}$.

Let $G^{\prime}$ be the plane subgraph of $G$ induced by the vertices of $C, P_{v_{1} a}$, and $P_{v_{k} c}$. The outer cycle of $G^{\prime}$ consists of edge $(a, c)$ and a path $P_{a c}$ between vertices $a$ and $c$.

Claim 2. The vertices of $P_{v_{1} a}$ and $P_{v_{k} c}$ can be placed on lines $\ell_{a}^{1}, \ell_{a}^{2}$ and $\ell_{c}$ such that in the resulting straight-line drawing of $G^{\prime}$, path $P_{a c}$ is represented by an $x$-monotone polygonal chain, and the inner faces of $G^{\prime}$ are star-shaped polygons.

The vertices of $P_{v_{1} a}$ will be placed on line $\ell_{a}^{1}$ between points $p$ and $q$ and on line $\ell_{a}^{2}$ above point $p_{a}$. The vertices of $P_{v_{k} c}$ will be placed on $\ell_{c}$ below $p_{c}$. In order to place 


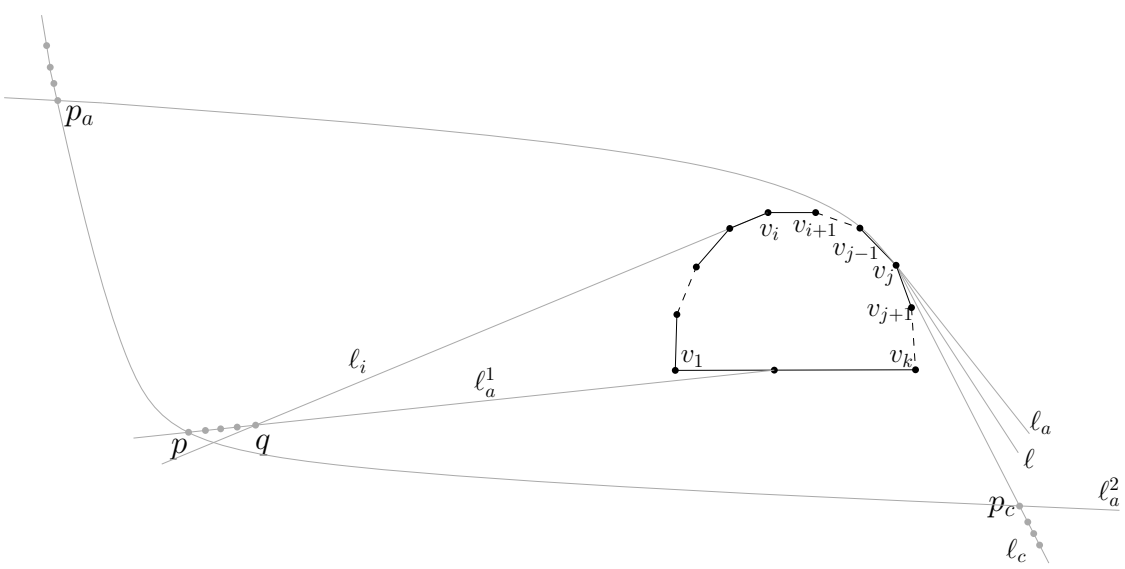

Fig. 3. Illustration for the proof of Theorem 2 For space reasons lines were shown by curves.

the vertices, we need to understand how the vertices of $P_{v_{1} a}$ are adjacent to vertices of $C$. As we travel on $P_{v_{1} a}$ from $v_{1}$ to $a$, we first meet all vertices adjacent to $v_{1}, \ldots, v_{i}$ and then all vertices adjacent to $v_{i+1}, \ldots, v_{j}$, since $G$ is a planar graph. Let $w$ be the first vertex of $P_{v_{1} a}$ adjacent to $v_{f}, i+1 \leq f \leq j$, and let $w^{\prime}$ be the vertex preceding $w$ on $P_{v_{1} a}$. We place vertices of $P_{v_{1} a}\left[v_{1}, w^{\prime}\right]$, in the order they appear in the path, on line $\ell_{a}^{1}$, between $q$ and $p$, in increasing distance from $v_{1}$. We place all vertices of $P_{v_{1} a}[w, a]$ on $\ell_{a}^{2}$ above $p_{a}$ in increasing distance from $p$. We draw the edges between the vertices of $C$ and $P_{v_{1} a}$. Notice that vertex $w$ might not exist, since it might happen that none of the vertices of $P_{v_{1} a}$ is adjacent to $v_{f}, i+1 \leq f \leq k$. In this case all vertices of $P_{v_{1} a}$ are placed on line $\ell_{a}^{1}$, between $q$ and $p$. In the following, we show that the constructed drawing is planar. Notice that the quadrilateral formed by the points $w, a, v_{j}, v_{i+1}$ is convex, by the choice of line $\ell_{a}^{2}$ and the positions of vertices $w$ and $a$ on it. Also, notice that the points of vertices $v_{i+1}, \ldots, v_{j}$ form a one-sided polygon with base segment $\overline{v_{i+1} v_{j}}$, which lies in the triangle $\triangle o v_{j} v_{i+1}$, where $o$ is the intersection of $\overline{v_{i+1} a}$ and $\overline{v_{j} w}$. Thus, by Fact 1 , the edges connecting $C\left[v_{i+1}, v_{j}\right]$ and $P_{v_{1} a}[w, a]$ do not cross each other. By applying Fact 1 , we can also prove that edges connecting $P_{v_{1} a}\left[v_{1}, w^{\prime}\right]$ with $C\left[v_{1}, v_{i}\right]$, cross neither each other, nor $\Gamma_{C}$. Recalling that vertices of $P_{v_{1} a}\left[v_{1}, w^{\prime}\right]$ can be also adjacent to $v_{k}$, we notice that these edges also do not cross $\Gamma_{C}$, by the choice of line $\ell_{a}^{1}$. Finally, path $P_{v_{1} a}$ is chordless, and therefore the current drawing is planar. Notice that the subpath of $P_{a, c}$ that has already been drawn is represented by an $x$-monotone chain. We next draw the vertices of $P_{v_{k} c}$. We observe that in the already constructed drawing path $P_{v_{1} a}$ taken together with edge $\left(v_{1}, v_{k}\right)$ is represented by an $x$-monotone chain, each point of which is visible from any point below the line $\ell_{a}^{2}$. This means that any point below line $\ell_{a}^{2}$, can be connected by a straight-line segment to the vertices $V\left(P_{v_{1} a}\right) \cup\left\{v_{k}\right\}$ without creating any crossing either with $P_{v_{1} a}$ or with $\left(v_{1}, v_{k}\right)$. We also notice that any of the vertices $v_{j}, \ldots, v_{k}$ can be connected to a point of $\ell_{c}$, without intersecting $\Gamma_{C}$. Recall that $p_{c}$ denotes the intersection point of $\ell_{c}$ and $\ell_{a}^{2}$. Thus we place the vertices of $P_{v_{k} c}$ on the line $\ell_{c}$, below $\ell_{a}^{2}$, in increasing distance from point $p_{c}$. Applying Fact 1 we can prove that the edges induced by $\left\{v_{j}, \ldots, v_{k}\right\} \cup V\left(P_{v_{k} c}\right)$ are 


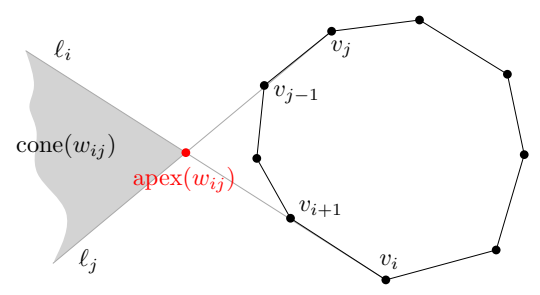

(a)

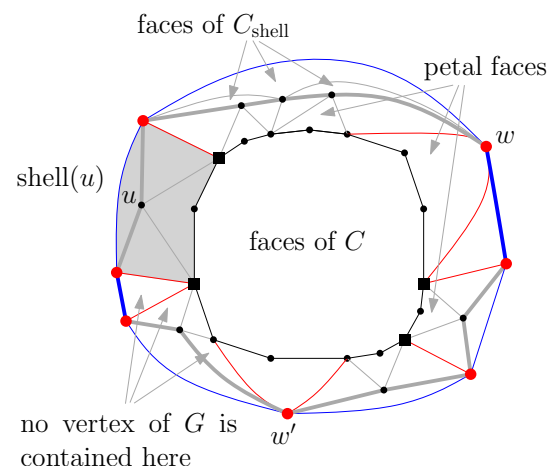

(b)

Fig. 4. (a) Vertex $w_{i, j}$ is the petal of $C$ with base $C\left[v_{i}, v_{j}\right]$. Point apex $\left(w_{i, j}\right)$ is red, region cone $\left(w_{i, j}\right)$ is gray. (b) Graph $G_{\text {shell }}$. Polygon $\Gamma_{C}$ is black. Cycle $C_{\text {shell }}$ is bold gray. Cycle $C_{\text {shell }}^{\prime}$ is blue. Graph $G_{\text {shell }}^{\prime}$ is comprised by blue, red and black edges. Vertices of $B$ are squares.

drawn without crossings. Edges between $P_{v_{k} c}$ and $P_{v_{1} a}$ cross neither $P_{v_{1} a}$, nor $\left(v_{1}, v_{k}\right)$ by the choice of lines $\ell_{c}$ and $\ell_{a}^{2}$.

We have constructed a planar straight-line drawing of $G^{\prime}$. We notice that path $P_{a c}$ is drawn as an $x$-monotone polygonal chain. We also notice that the faces of $G^{\prime}$, created when placing vertices of $P_{v_{1} a}$ (resp. $P_{v_{k} c}$ ) are star-shaped and have their kernels arbitrarily close to the vertices of $P_{v_{1} a}$ (resp. $P_{v_{k} c}$ ).

Notice that vertex $b$ is possibly adjacent to some of the vertices of $P_{a c}$. Thus, placing $b$ at an appropriate distance above $P_{a c}$, the edges between $b$ and $P_{a c}$ can be drawn straight-line without intersecting $P_{a c}$ and therefore no other edge of $G^{\prime}$. The faces created when placing $b$ are star-shaped and have their kernels arbitrarily close to $b$. We finally apply Theorem 1

\section{Main Theorem}

Let $G$ be a maximal plane graph and $C$ be a strictly internal simple outerchordless cycle of $G$, represented by an arbitrary convex polygon $\Gamma_{C}$ in the plane. In Theorem 3 we prove that it is still true that if each petal of $C$ is realizable, then $\Gamma_{C}$ is extendable. Before stating and proving Theorem 3 , we introduce notation that will be used through this section.

Recall that $v_{1}, \ldots, v_{k}$ denote the vertices of $C$. Let $w_{i, j}$ be an outermost petal of $C$ in $G$. Let $\ell_{i}$ (resp. $\ell_{j}$ ) be a half-line with the slope of edge $\left(v_{i}, v_{i+1}\right)\left(\operatorname{resp} .\left(v_{j-1}, v_{j}\right)\right.$ ) originating at $v_{i}$ (resp. $v_{j}$ ) (see Figure 4(a). Since $w_{i, j}$ is realizable, lines $\ell_{i}$ and $\ell_{j}$ intersect. Denote by $\operatorname{apex}\left(w_{i, j}\right)$ their intersection point and by cone $\left(w_{i, j}\right)$ the subset of $\mathbb{R}^{2}$ that is obtained by the intersection of the half-planes defined by $\ell_{i}$ and $\ell_{j}$, not containing $\Gamma_{C}$. It is clear that any internal point of cone $\left(w_{i, j}\right)$ is appropriate to draw $w_{i, j}$ so that the plane subgraph of $G$ induced by $V(C) \cup\left\{w_{i, j}\right\}$ is crossing-free. For consistency, we also define cone $(w)$ and $\operatorname{apex}(w)$ of an outer stamen $w$ of $C$ as follows. 


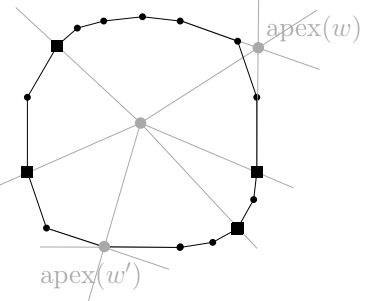

(a)

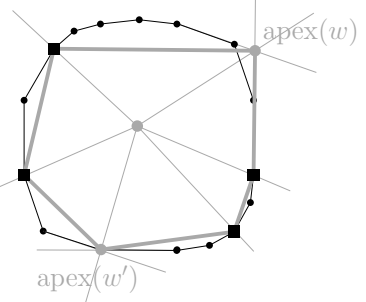

(b)

Fig. 5. Construction of drawing of graph $G_{\text {shell }}$ shown in Figure 4(b) (a) Apex points are gray, points of $B$ are black squares. (b) Polygon $\Pi$ is gray, lines $\left\{\ell(w) \mid w \in S \cap C_{\text {shell }}^{\prime}\right\}$ are dashed.

Assume that $w$ is adjacent to $v_{i} \in C$. Then $\operatorname{cone}(w) \subset \mathbb{R}^{2}$ is the union of the halfplanes defined by lines of edges $\left(v_{i-1}, v_{i}\right)$ and $\left(v_{i}, v_{i+1}\right)$, that do not contain $\Gamma_{C}$. We $\operatorname{set} \operatorname{apex}(w)=v_{i}$.

Let $P$ (resp. $S$ ) denote the set of outermost petals (resp. stamens) of $C$ in $G$. By Lemma 1 there exists a cycle $C_{\text {shell }}$ in $G$ that contains exactly $P \cup S$. Let $G_{\text {shell }}$ denote the plane subgraph of $G$ induced by the vertices of $C$ and $C_{\text {shell }}$. (Figure 4(b)). Let $C_{\text {shell }}^{\prime}$ denote the outer cycle of $G_{\text {shell }}$. We denote the graph consisting of $C, C_{\text {shell }}^{\prime}$ and edges between them by $G_{\text {shell }}^{\prime}$. Each petal or stamen of $C$, say $w$, that belongs to $C_{\text {shell }}$ but not to $C_{\text {shell }}^{\prime}$, belongs to a face of $G_{\text {shell }}^{\prime}$. We denote this face by $\operatorname{shell}(w)$. We categorize the faces of $G_{\text {shell }}$ as follows. The faces that lie inside cycle $C$ are called faces of $C$. The faces that are bounded only by $C_{\text {shell }}$ and its chords, are called faces of $C_{\text {shell }}$. Notice that each face of $C_{\text {shell }}$ is a triangle. Notice that a face of $G_{\text {shell }}$ that is comprised by two consecutive edges adjacent to the same vertex of $C$ (not belonging to $C$ ), is a triangle, and contains no vertex of $G \backslash G_{\text {shell }}$, since both facts would imply that the taken edges are not consecutive. Finally, faces bounded by a subpath of $C$ and two edges adjacent to the same petal, are called petal faces. The plane subgraph of $G$ inside a petal face is triangulated and does not have a chord connecting two vertices of its outer face, and therefore is triconnected. Thus we have the following

Observation 1. Each vertex of $G \backslash G_{\text {shell }}$ either lies in a face of $C$, or in a face that is

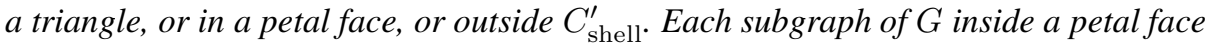
is triconnected.

Theorem 3. Let $G$ be a maximal plane graph and let $C$ be a strictly internal simple outerchordless cycle of $G$, represented by a convex polygon $\Gamma_{C}$ in the plane. $\Gamma_{C}$ is extendable to a straight-line drawing of $G$ if and only if each petal of $C$ is realizable.

Proof. The condition that each petal of $C$ is realizable is clearly necessary. Next we show that it is also sufficient.

We first show how to draw the graph $G_{\text {shell }}^{\prime}$. Afterward we complete it to a drawing of $G_{\text {shell }}$. Our target is to represent $C_{\text {shell }}^{\prime}$ as a one-sided polygon, so that Theorem 2 can be applied for the rest of $G$ that lies outside $C_{\text {shell }}^{\prime}$. We first decide which edge of $C_{\text {shell }}^{\prime}$ to "stretch", i.e., which edge will serve as base edge of the one-sided polygon 


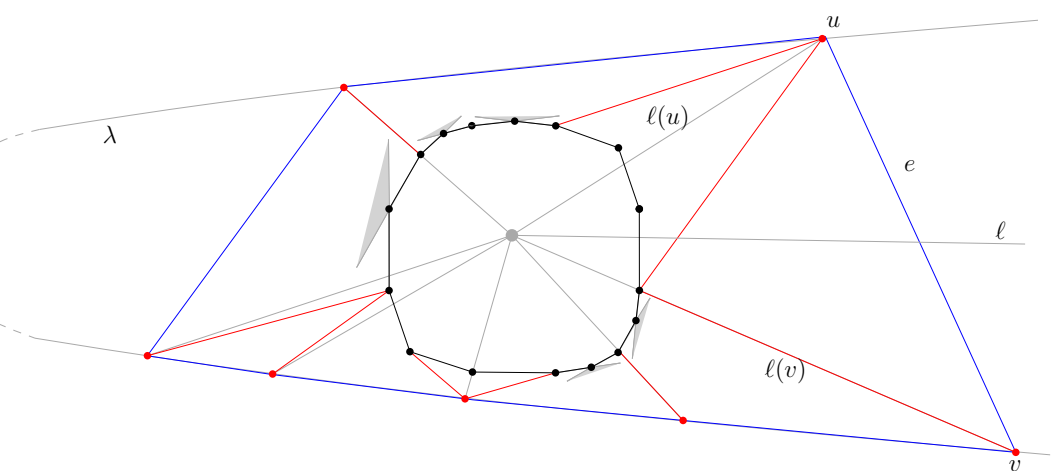

Fig. 6. Construction of Case 1. Corresponding $G_{\text {shell }}$ is shown in Figure 4(b)

for representing $C_{\text {shell }}^{\prime}$. In order to be able to apply Theorem 2 , this one-sided polygon should be such that each petal of $C_{\text {shell }}^{\prime}$ is realizable. Thus we choose the base edge $e$ of $C_{\text {shell }}^{\prime}$ as follows. If $C_{\text {shell }}^{\prime}$ contains an edge on the outer face of $G$, we choose $e$ to be this edge. Otherwise, we choose an edge $e$, such that at least one of the end vertices of $e$ is adjacent to an outermost petal of $C_{\text {shell }}^{\prime}$ in $G$. Such a choice of $e$ ensures that each petal of $C_{\text {shell }}^{\prime}$ is realizable.

Claim 3. Polygon $\Gamma_{C}$ can be extended to a straight-line drawing of graph $G_{\text {shell }}^{\prime}$, such that its outer face $C_{\text {shell }}^{\prime}$ is represented by a one-sided polygon with base edge e. Moreover, $C_{\text {shell }}^{\prime}$ contains in its interior all points of $\left\{\operatorname{apex}(w) \mid w \in C_{\text {shell }}\right\}$.

Recall that $P$ (resp. $S$ ) denotes the set of outermost petals (resp. stamens) of $C$ in $G$. Let $B$ denote the set of vertices of $C$, to which stamens $S \cap C_{\text {shell }}^{\prime}$ are adjacent (refer to Figure 4(b)]. By construction of the apex points, the set $\{\operatorname{apex}(w) \mid w \in$ $\left.P \cap C_{\text {shell }}^{\prime}\right\} \cup B$ is in convex position, and we denote by $\Pi$ its convex hull. Polygon $\Pi$ may be degenerate, and may contain only a single vertex or a single edge. We treat these cases separately to complete the construction of the drawing of the graph $G_{\text {shell }}^{\prime}$. Next, we explain the construction in the non-degenerate case; the degenerate cases are covered in the full version of this paper [7].

Let $p$ be a point inside $\Pi$. Let $\ell(w)$ denote a half-line from $p$ through $w$, where $w$ is a vertex of $\Pi$. If we order the constructed half-lines around $p$, any two consecutive lines have between them an angle less than $\pi$. If $w \in B$, we substitute $\ell(w)$ by the same number of slightly rotated lines as the number of stamens of $C_{\text {shell }}^{\prime}$ adjacent to $w$, without destroying the order (refer to Figure 5(b)]. Thus, for each $w \in C_{\text {shell }}^{\prime}$, a line $\ell(w)$ is defined. Notice that, for any $w \in P \cap C_{\text {shell }}^{\prime}$, line $\ell(w)$ passes through apex $(w)$, and the infinite part of $\ell(w)$ lies in cone $(w)$. Thus, for any position of $w$ on a point of $\ell(w) \cap \operatorname{cone}(w)$, edges between $C$ and $w$ do not cross $\Gamma_{C}$. For a stamen $w \in S \cap C_{\text {shell }}^{\prime}$, line $\ell(w)$ crosses cone $(w)$ very close to apex $(w)$, and its infinite part lies in cone $(w)$. Thus, similarly, for any position of $w$ on a point of $\ell(w) \cap \operatorname{cone}(w)$, edges between $C$ and $w$ do not cross $\Gamma_{C}$.

Recall that $e=(u, v)$ is the edge of $C_{\text {shell }}^{\prime}$ that we have decided to "stretch". Recall also that $\ell(u)$ and $\ell(v)$ are consecutive in the sequence of half-lines we have 
constructed. Let $\kappa$ be a circle around $\Gamma_{C}$ that contains in the interior the polygon $\Pi$ and the set of points $\left\{\operatorname{apex}(w) \mid w \in C_{\text {shell }}\right\}$. Let $\ell$ be a half-line bisecting the angle between $\ell(u)$ and $\ell(v)$ (refer to Figure 6). Let $\lambda$ be a parabola with $\ell$ as axis of symmetry and the center of $\kappa$ as focus. We position and parametrize $\lambda$ such that it does not cross $\ell$ and $\kappa$.

With this placement of $\lambda$, each half-line $\ell(w), w \in \Pi$, crosses $\lambda$, moreover, intersections with $\ell(u)$ and $\ell(v)$ are on different branches of $\lambda$ and appear last on them as we walk on $\lambda$ from its origin to infinity. Let $\Pi^{\prime}$ be the convex polygon comprised by the intersection points of lines $\left\{\ell(w): w \in V\left(C_{\text {shell }}^{\prime}\right)\right\}$ with $\lambda$. We make $\lambda$ large enough, so that the polygon $\Pi^{\prime}$ still contains the circle $\kappa$ in the interior. As a results, for each $w \in C$, cone $(w) \cap \Pi_{\text {in }}^{\prime} \neq \emptyset$, where $\Pi_{\text {in }}^{\prime}$ denotes the interior of $\Pi^{\prime}$. This concludes the proof of the claim in the non-degenerate case.

Let $\Gamma_{\text {shell }}^{\prime}$ be the constructed drawing of $G_{\text {shell }}^{\prime}$. Recall that each petal or stamen $w$ of $C$, that does not belong to $C_{\text {shell }}^{\prime}$, lies in a face of $G_{\text {shell }}^{\prime}$, denoted by $\operatorname{shell}(w)$. Let $\Gamma_{\operatorname{shell}(w)}$ denote the polygon representing face shell $(w)$ in $\Gamma_{\text {shell }}^{\prime}$. By construction, $\operatorname{cone}(w) \cap \Gamma_{\text {shell }(w)} \neq \emptyset$. We next explain how to extend the drawing of $G_{\text {shell }}^{\prime}$ to the drawing of $G_{\text {shell }}$. For each edge $(u, v)$ of $C_{\text {shell }}^{\prime}$, we add a convex curve, lying close enough to this edge inside $\Gamma_{\text {shell }}^{\prime}$. Let $\mu$ be the union of these curves for all edges of $C_{\text {shell }}^{\prime}$. We notice that we can place them so close to $C_{\text {shell }}^{\prime}$ that all the points of $\{\operatorname{apex}(w) \mid w \in C\}$ are still in the interior of $\mu$. Thus $\mu$ is intersected by all the sets cone $(w)$, for each $w \in C$. We place each vertex $w$ of $C_{\text {shell }} \backslash C_{\text {shell }}^{\prime}$ on $\mu \cap \operatorname{cone}(w)$ in the order they appear in $C_{\text {shell }}$. Since all edges induced by $C_{\text {shell }}$ lie outside of $C_{\text {shell }}$, and both end points of such an edge are placed on a single convex curve, they can be drawn straight without intersecting each other, or other edges of $G_{\text {shell }}$. Thus, we have constructed a planar extension of $\Gamma_{C}$ to a drawing of $G_{\text {shell }}$, call it $\Gamma_{\text {shell }}$.

Recall the definitions of faces of $C$, faces of $C_{\text {shell }}$ and petal faces from the beginning of this section. The faces of $C$ appear in $\Gamma_{\text {shell }}$ as convex polygons. The faces of $C_{\text {shell }}$ are triangles, and the petal faces of $G_{\text {shell }}$ are star-shapes whose kernel is close to the corresponding petal. By Observation 1 , each vertex of $G \backslash G_{\text {shell }}$ either lies in a face of $C$, or in a face that is a triangle, or in a petal face, or outside $C_{\text {shell }}^{\prime}$. Moreover a subgraph of $G$ inside a petal face is triconnected. Thus, by multiple applications of Theorem 1 , we can extend the drawing of $G_{\text {shell }}$ to a straight-line planar drawing of the subgraph of $G$ inside $C_{\text {shell }}^{\prime}$.

Finally, notice that in the constructed drawing of $G_{\text {shell }}$ each petal of its outer cycle, i.e. $C_{\text {shell }}^{\prime}$, is realizable. This is by the choice of edge $e$. Moreover, by construction of $G_{\text {shell }}, C_{\text {shell }}^{\prime}$ has no outer chords. In case $C_{\text {shell }}^{\prime}$ is not strictly internal, we apply Proposition 1 to construct a maximal plane graph $G^{\prime}$, such that $G$ is a plane subgraph of $G^{\prime}, C_{\text {shell }}^{\prime}$ is a strictly internal outerchordless cycle of $G^{\prime}$ and each petal of $C_{\text {shell }}^{\prime}$ is realizable. Then, we apply Theorem 2 , to complete the drawing of $G^{\prime}$, lying outside $C_{\text {shell }}^{\prime}$. Finally, we remove the edges of $G^{\prime}$ that do not belong to $G$.

We conclude with the following general statement, that follows from Proposition 1 , Theorem 3 and one of the known algorithms that constructs drawing of a planar graph with a prescribed outer face (e.g. [4]10] or Theorem 1].

Corollary 1. Let $G$ be a plane graph and $H$ be a biconnected plane subgraph of $G$. Let $\Gamma_{H}$ be a straight-line convex drawing of $\Gamma_{H}$. $\Gamma_{H}$ is extendable to a planar straight-line 
drawing of $G$ if and only if the outer cycle of $H$ is outerchordless and each petal of the outer cycle of $H$ is realizable.

\section{Conclusions}

In this paper, we have studied the problem of extending a given convex drawing of a cycle of a plane graph $G$ to a planar straight-line drawing of $G$. We characterized the cases when this is possible in terms of two simple necessary conditions, which we proved to also be sufficient. We note that it is easy to test whether the necessary conditions are satisfied in linear time. It is readily seen that our proof of existence of the extension is constructive and can be carried out in linear time. As an extension of our research it would be interesting to investigate whether more envolved necessary conditions are sufficient for more general shape of a cycle, for instance a star-shaped polygon.

Acknowledgments. M.N. received financial support by the Concept for the Future of KIT. I.R. was supported by a fellowship within the Postdoc-Program of the German Academic Exchange Service (DAAD). Part of this work was done within GRADR EUROGIGA project no. 10-EuroGIGA-OP-003.

\section{References}

1. Angelini, P., Di Battista, G., Frati, F., Jelínek, V., Kratochvíl, J., Patrignani, M., Rutter, I.: Testing planarity of partially embedded graphs. In: 21st Annual ACM-SIAM Symposium on Discrete Algorithms (SODA 2010), pp. 202-221. SIAM (2010)

2. Avis, D.: Generating rooted triangulations without repetitions. Algorithmica 16, 618-632 (1996)

3. Chambers, E.W., Eppstein, D., Goodrich, M.T., Löffler, M.: Drawing graphs in the plane with a prescribed outer face and polynomial area. Journal of Graph Algorithms and Applications 16(2), 243-259 (2012)

4. Duncan, C.A., Goodrich, M.T., Kobourov, S.G.: Planar drawings of higher-genus graphs. Journal of Graph Algorithms and Applications 15(1), 7-32 (2011)

5. Hong, S.-H., Nagamochi, H.: Convex drawings of graphs with non-convex boundary constraints. Discrete Applied Mathematics 156(12), 2368-2380 (2008)

6. Jelínek, V., Kratochvíl, J., Rutter, I.: A Kuratowski-type theorem for planarity of partially embedded graphs. Computational Geometry Theory \& Applications 46(4), 466-492 (2013)

7. Mchedlidze, T., Nöllenburg, M., Rutter, I.: Drawing planar graphs with a prescribed inner face. CoRR, abs/1308.3370 (2013)

8. Pach, J., Wenger, R.: Embedding planar graphs at fixed vertex locations. In: Whitesides, S.H. (ed.) GD 1998. LNCS, vol. 1547, pp. 263-274. Springer, Heidelberg (1999)

9. Patrignani, M.: On extending a partial straight-line drawing. In: Healy, P., Nikolov, N.S. (eds.) GD 2005. LNCS, vol. 3843, pp. 380-385. Springer, Heidelberg (2006)

10. Tutte, W.T.: How to draw a graph. Proc. London Math. Soc. 13(3), 743-768 (1963) 